\title{
Altered diversity and composition of the gut microbiome in patients with cervical cancer
}

\author{
Zhongqiu Wang ${ }^{1 \dagger}$, Qingxin Wang ${ }^{1 \dagger}$, Jing Zhao ${ }^{1 \dagger}$, Linlin Gong ${ }^{1}$, Yan Zhang ${ }^{2}$, Xia Wang ${ }^{3}$ and Zhiyong Yuan ${ }^{1 *}$ (]
}

\begin{abstract}
Gut microbiota have been implicated in the development of many human diseases, including both digestive diseases and non-digestive diseases. In this study, we investigated whether the gut bacteria differed in cervical cancer (CCa) patients compared with healthy controls by 165 rRNA sequencing analysis. Subjects including eight CCa and five healthy controls were included. Microbiota profiles in fecal DNA were characterized by PCR amplification of the $16 \mathrm{~S}$ rRNA V4 variable region and deep sequencing using the Illumina HiSeq platform. The CCa-associated gut microbiota had an increasing trend in alpha diversity, although statistical significance was not reached. Inter-group variability in community structure by beta diversity analysis showed a clear separation between cancer patients and healthy controls. Gut microbiota profiles were different between patients and controls; namely, the proportions of Proteobacteria phylum was notably higher in patients with CCa $(\rho=0.012)$. Seven genera differentiated significantly in relative abundance between CCa and controls (all $\rho<0.05$ ), including Escherichia-Shigella, Roseburia, Pseudomonas, Lachnoclostridium, Lachnospiraceae_UCG-004, Dorea and Succinivibrio. The characteristic microbiome in CCa patients was also identified by linear discriminant analysis effect size (LEfSe). The phylum Proteobacteria, and the genus Parabacteroides, Escherichia_Shigells and Roseburia may provide novel potential biomarkers for CCa. Taken together, this is the first study on gut microbiota in patients with CCa, and demonstrated the significantly altered diversity and composition.
\end{abstract}

Keywords: Cervical cancer, Gut microbiota, $16 \mathrm{~S}$ rRNA, Deep sequencing

\section{Introduction}

Cervical cancer is the fourth most common cause of cancer-related deaths in women worldwide, and the most common gynecological neoplasia in developing countries. Current literature reports that globally, there are approximately 500,000 new cases of cervical cancer, and more than 270,000 deaths annually (Basu et al. 2017). In recent decades, etiological factors such as infection with high-risk papilloma viruses (HPV) have been well established in cervical cancer. However, understanding of carcinogenesis is still insufficient, and the evidence suggests

\footnotetext{
*Correspondence: dryuantj@163.com

†Zhongqiu Wang, Qingxin Wang and Jing Zhao contributed equally to this work

${ }^{1}$ Department of Radiation Oncology, Key Laboratory of Cancer Prevention and Therapy, Tianjin's Clinical Research Center for Cancer, Tianjin Medical University Cancer Institute \& Hospital, National Clinical Research Center for Cancer, West Huanhu Road, West River District, Tianjin 300060, China

Full list of author information is available at the end of the article
}

that many other host variations are important in the development of cervical cancer (Martin et al. 2007).

The human gastrointestinal tract carries about $10^{14}$ microbes. The genetic content of these microbial communities is approximately 100 times greater than seen in human genes (Human microbiome project consortium 2012). They co-exist with their hosts as a super-organism in a mutualistic manner and play fundamental roles in human health and disease. For example, emerging evidence shows that the intestinal microbiota regulates the host's metabolism and also stimulates and renews epithelial cells. In addition, the intestinal microbiota will influence the development and maturation of the nervous and immune systems (Vrieze et al. 2013; Ursell et al. 2014; Dinan and Cryan 2017; Partida-Rodríguez et al. 2017). In return, individual signature contributes to differences in the gut microbiota. The structure of the gut microbial community changes constantly according to various external variables such as age, sex, stress, probiotic or antibiotic usage and genetic background (Sommer and 
Bäckhed 2013). Kozik et al. (2017) has demonstrated that the mouse fecal microbiome is partially shaped by factors such as sex, age and TNF production. These effects correlate with the severity of the animals' colitis.

While several studies have investigated the role of the gut microbiota in the etiology of digestive disease: including inflammatory bowel disease (Pascal et al. 2017), hepatitis (Heidrich et al. 2018), and colorectal cancer (Wong et al. 2017), the effects on the development of cancer in other parts of the body have been limited. With the advent of next-generation $16 \mathrm{~S}$ rRNA gene deep sequencing, the microbiome can now be characterized in a depth and detail that was not previously available. In this study, through $16 \mathrm{~S}$ rRNA gene sequencing, we identified specific microbial signatures in patients with cervical cancer and sought to elucidate potential biomarkers or underlying mechanism how the microbiota may influence the pathogenesis of cervical cancer.

\section{Materials and methods}

\section{Study participants}

A total of eight patients with cervical cancer (CCa), who had not received any treatments were recruited in our department between June 2015 to January 2016. The detailed clinical parameters are shown in Table 1. Fecal samples were obtained 1 day after the patient was pathology confirmed. None of the patients had either used antibiotics or probiotics within 2 months or taken proton pump inhibitors within at least 2 weeks before sample collection. Exclusion criteria also included factors known to impact the intestinal microbiota, such as inflammatory bowel disease, existed abnormal bowel symptoms (e.g. abdominal pain, tenesmus, fecal incontinence or diarrhea), and other types of cancer (Pascal et al. 2017; Wong et al. 2017). Another five age-matched healthy female controls (HCs) were also enrolled. Detail informed written consent was obtained from all participants. The study was conducted according to the Declaration of Helsinki and approved by the Medical Ethics Committee of Tianjin Medical University Cancer Institute \& Hospital.

\section{Sample collection and DNA extraction}

Fecal samples were freshly collected by participants,. deposited in a sterile container containing RNAlater, and stored at $-20{ }^{\circ} \mathrm{C}$. The QIAamp DNA Micro Kit (QIAGEN, Hilden, Germany) was used to extract microbial metagenomic DNA from $200 \mathrm{mg}$ of each sample.

\section{S rRNA gene sequencing}

PCR reactions were carried out using Phusion ${ }^{\circledR}$ HighFidelity PCR Master Mix (New England Biolabs). 16S rRNA genes of distinct V4 hypervariable region were amplified used bar-coded specific primer (515F-806R) as
Table 1 Patient characteristics

\begin{tabular}{lc}
\hline Characteristics & Values \\
\hline Age (years) & $59.5($ range $37-72)$ \\
Karnofsky performance score $\geqq 70$ & $8(100 \%)$ \\
FIGO stage & \\
II & $6(75.0 \%)$ \\
III & $2(25.0 \%)$ \\
Histology & \\
Squamous carcinoma & $7(87.5 \%)$ \\
Adenocarcinoma & $1(12.5 \%)$ \\
Differentiation & \\
Well/moderate & $5(62.5 \%)$ \\
Poor & $3(37.5 \%)$ \\
Diameter of tumor (cm) & \\
$\geq 4$ & $3(37.5 \%)$ \\
$<4$ & $5(62.5 \%)$ \\
Vaginal infiltration & \\
Presented & $4(50.0 \%)$ \\
None & $4(50.0 \%)$ \\
Lymph node metastasis & \\
Presented & $5(62.5 \%)$ \\
None & $3(37.5 \%)$ \\
\hline
\end{tabular}

previously described prior to sequencing (Caporaso et al. 2011). After PCR products were purified, sequencing libraries were generated using TruSeq ${ }^{\circledR}$ DNA PCR-Free Sample Preparation Kit (Illumina, USA), and then the index codes were attached. Quality of library was determined on the Qubit@ 2.0 Fluorometer (Thermo Scientific) and Agilent Bioanalyzer 2100 system followed by sequencing on the IlluminaHiSeq 2500 platform and generation of the paired-end reads ( $250 \mathrm{bp})$.

\section{Bioinformatics analysis}

The paired-end reads obtained was assigned to samples based on barcode. Barcode and primer sequence were cut off and truncated reads were merged using FLASH (V1.2.7) (Magoč and Salzberg 2011). Quality filtering on the raw tags was performed with QIIME qualitycontrolled process (V1.7.0) as previously described (Caporaso et al. 2017). To detect chimera sequences, the clean tags were blasted with the reference database (http://drive5.com/uchime/uchime_download.html) using the UCHIME algorithm (http://www.drive5.com/ usearch/manual/uchime_algo.html) (Edgar et al. 2011). The effective tags were obtained after remove of the chimera sequences (Haas et al. 2011).

Sequences analysis was carried out by Uparse software (V7.0.1001) (Edgar 2013). Sequences at 97\% were assigned to the same operational taxonomic units (OTUs). In order to annotate taxonomic information, 
sequence for each OTU was screened using GreenGene Database (DeSantis et al. 2006) based on RDP classifier algorithm (V2.2) (Wang et al. 2007). At last, multiple sequence alignment was conducted using the MUSCLE software (V3.8.31), to analyze the phylogenetic relationship and the dominant species (Edgar 2004).

\section{Data analysis}

Alpha diversity was calculated using Chao1 index, Shannon index, et al. with QIIME (V1.7.0) and displayed with $\mathrm{R}$ software (V2.15.3). Beta diversity was measured using both weighted and unweighted unifrac distance metrics by QIIME software (V1.7.0). Principal coordinate analysis (PCoA) was expressed by WGCNA package, ggplot2 packages and stat package in $\mathrm{R}$ software (V2.15.3). $\rho<0.05$ was taken as statistical significance.

\section{Results}

\section{Richness and diversity analysis}

In total, 783,660 reads, with an average of 64,361 reads per sample were generated after initial quality filtering (median read length $=253 \mathrm{bp}$ ). The total number of OTUs was 6665 at more than $97 \%$ similarity level. The alpha diversity indices, including observed species, Chao1, Shannon index, phylogenetic diversity (PD) whole tree, and abundance-based coverage estimator (ACE), were calculated for each data set (Table 2). Chao- 1 is a measure of total richness and is particularly useful because of a valid variance which can be used to calculate confidence intervals (Chao 1987; Wang et al. 2005). The Shannon index reflects species numbers and evenness of species abundance (Guinane et al. 2013). PD whole tree reflects the sum of all branch-lengths on the constructed phylogenetic tree from all taxa (Goedert et al. 2015). Our results showed that the fecal microbiota of CCa patients had overall higher alpha diversity than those of the healthy controls, although no significant difference was observed by $t$-test (Fig. $1 \mathrm{a}-\mathrm{e}$ ).

The species accumulation curve reached asymptotic values in each sample, revealing that the sampling effort was sufficient to figure out the most genera present (Fig. 1f). The rarefaction curve reached a summit in each sample, indicating that the sequencing depth was sufficient to detect all the genera within each sample and beneficial to capture the microbial diversity (Fig. 1g).

Beta diversity was first evaluated with weightedUniFrac analysis (Fig. 2a, b, $\rho=0.004$ ). UniFrac-based PCoA provided an entire comparison of microbial communities, and showed that the $\mathrm{CCa}$ cohort and the $\mathrm{HC}$ cohort show clear separation (Fig. 2c). Furthermore, non-metric multi-dimensional scaling (NMDS) showed that the fecal microbiota of CCa group was distinct from the $\mathrm{HC}$ group (Fig. 2d ANOSIM, $\mathrm{R}=0.6224, \rho=0.001$; MRPP, $A=0.07559$, observed-delta $=0.5686$, expecteddelta $=0.6151, \rho=0.004)$. NMDS analysis based on Sorensen (Bray-Curtis) distance indicated noted differences in microbial communities at the second MDS between $\mathrm{CCa}$ group and $\mathrm{HC}$ group (MDS1, $\rho>0.05$; MDS2, $\rho=0.0016$; Mann-Whitney U test, Fig. 2e, f).

\section{Comparative analysis of the gut microbial composition of patients with cervical cancer and healthy controls} Assigned sequence reads were used to assess differences in taxonomic abundances between $\mathrm{CCa}$ and $\mathrm{HCs}$ at various levels. At the phylum level, Bacteroidetes was the most abundant, contributing $54.19 \%$ and $51.96 \%$ of the gut microbiota in CCa group and $\mathrm{HC}$ group respectively, followed by Firmicutes (29.55\% and $16.00 \%$ respectively) and Proteobacteria (38.28\% and 7.9\% respectively) (Fig. 3a). Microbial compositions showed high

Table 2 Estimation of diversity at the $97 \%$ similarity level within each data set

\begin{tabular}{|c|c|c|c|c|}
\hline Sample & Shannon index & Simpson index & Chao1 richness & Good's coverage \\
\hline $\mathrm{HC} 1$ & 5.079 & 0.936 & 461.250 & 0.997 \\
\hline $\mathrm{HC} 2$ & 5.561 & 0.944 & 643.775 & 0.996 \\
\hline $\mathrm{HC} 3$ & 5.045 & 0.941 & 342.021 & 0.998 \\
\hline $\mathrm{HC} 4$ & 4.172 & 0.830 & 658.015 & 0.994 \\
\hline $\mathrm{HC} 5$ & 4.789 & 0.902 & 284.857 & 0.999 \\
\hline CCa1 & 6.023 & 0.966 & 552.016 & 0.996 \\
\hline $\mathrm{CCa} 2$ & 5.515 & 0.930 & 521.060 & 0.997 \\
\hline CCa3 & 5.912 & 0.962 & 547.294 & 0.997 \\
\hline CCa4 & 5.810 & 0.955 & 567.056 & 0.996 \\
\hline CCa5 & 5.999 & 0.961 & 516.623 & 0.997 \\
\hline CCa6 & 4.355 & 0.843 & 497.978 & 0.997 \\
\hline $\mathrm{CCa} 7$ & 5.123 & 0.888 & 530.344 & 0.997 \\
\hline CCa8 & 5.633 & 0.951 & 620.238 & 0.996 \\
\hline
\end{tabular}




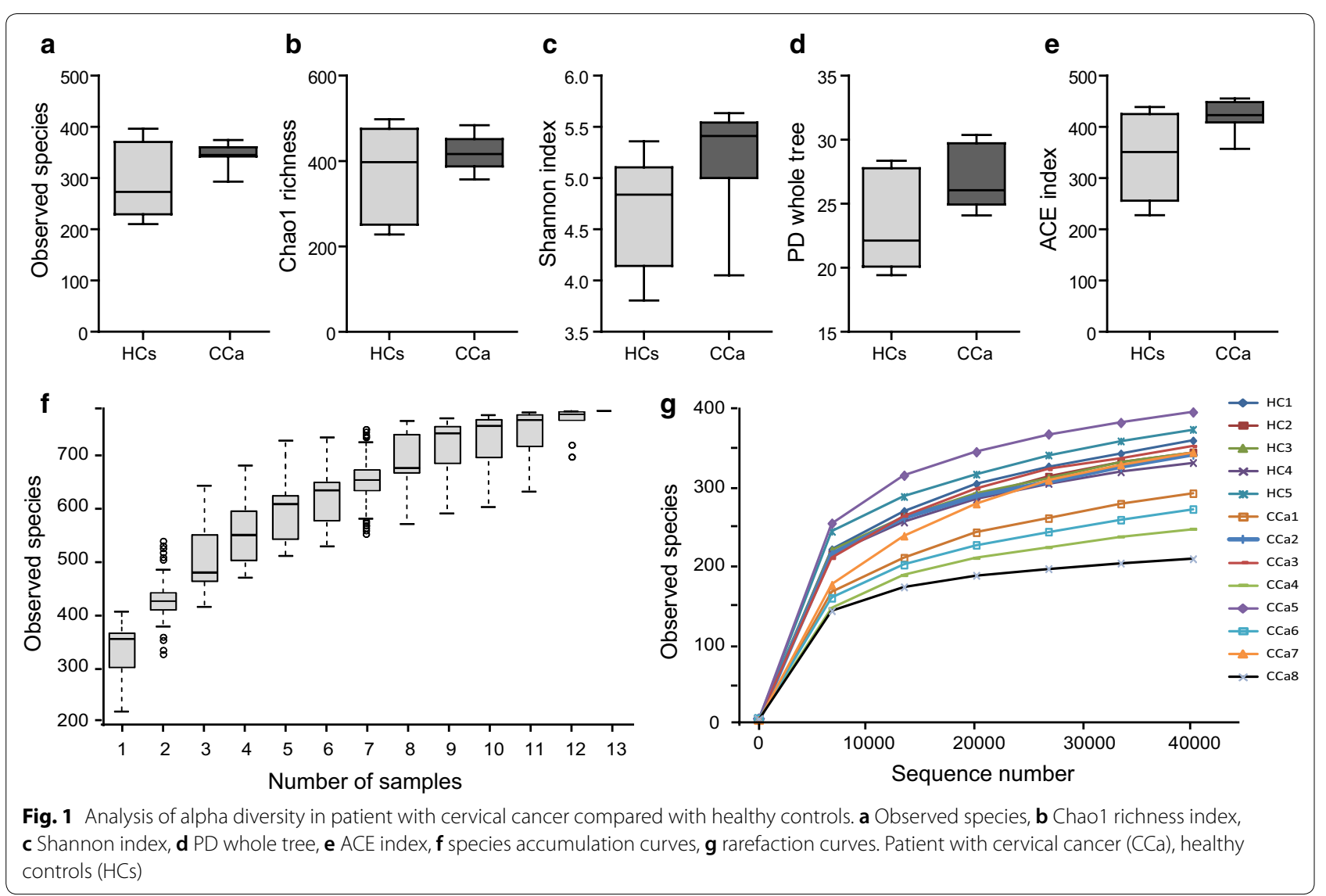

inter-individual variability. For example, Bacteroidetes accounted for $34.08-68.82 \%$, Firmicutes $18.57-47.24 \%$, and Proteobacteria 3.79-25.95\% among all the individuals. The relative abundance of Proteobacteria in CCa patients was significantly higher $(\rho=0.012)$, while overabundance of Bacteroidetes and Firmicutes did not reach statistical significance (Fig. 3b).

At the class level, a significant increase was observed in the abundance of Gammaproteobacteria in CCa group (Fig. 4a, $\rho=0.009$ ). Moreover, upon closer examination of taxonomic data, we noted that $\mathrm{CCa}$ group were enriched with order Enterobacteriales $(\rho=0.008)$, Aeromonadales $(\rho<0.001)$, Oceanospirillales $(\rho=0.020)$ and Alteromonadales $(\rho=0.001)$ from the Gammaproteobacteria class (Fig. 4b).

At the family level, eight families were presented at significantly altered proportions $(\rho<0.05)$ in $\mathrm{CCa}$ patients compared to HCs. Five families increased including Enterobacteriaceae $\quad(\rho=0.008), \quad$ Pseudomonadaceae $(\rho=0.019)$, Succinivibrionaceae $(\rho<0.001)$ and Halomonadaceae $(\rho=0.008)$. Whereas Acidaminococcaceae were decreased $(\rho=0.019)$.

Genus-level analysis was more informative (Fig. 4c and Table 3). The data revealed that genus Phascolarctobacterium $(\rho=0.036)$ was notably overabundant in
HCs, possibly inflating the high Firmicutes abundance at the phylum level. While genera Escherichia-Shigella $(\rho=0.030)$ and Roseburia $(\rho=0.049)$ were more abundant in CCa group. Other minor genera that were also significantly enriched in CCa patients were Pseudomonas $(\rho=0.018), \quad$ Lachnoclostridium $(\rho=0.018), \quad$ Lachnospiraceae_UCG-004 $(\rho=0.018)$, Dorea $(\rho=0.026)$, and Succinivibrio $(\rho<0.001)$.

\section{Comparative analysis of the gut microbial taxa}

between patients with cervical cancer and healthy controls We further compared taxa in the CCa vs. HC groups by discriminant analysis effect size (LEfSe). The LEfSe method is used to discover high-dimensional biomarker. The linear discriminant analysis (LDA) model identifies differently abundant taxa between groups and estimates the effect size of each significantly different taxon (Segata et al. 2011).

LEfSe analysis revealed that the phylum Proteobacteria, and the genus Parabacteroides, Escherichia_Shigells, and Roseburia were all significantly more abundant in the fecal samples from the patients with $\mathrm{CCa}$ and conversely, significantly less abundant in the fecal samples from healthy controls (Fig. 5a). 


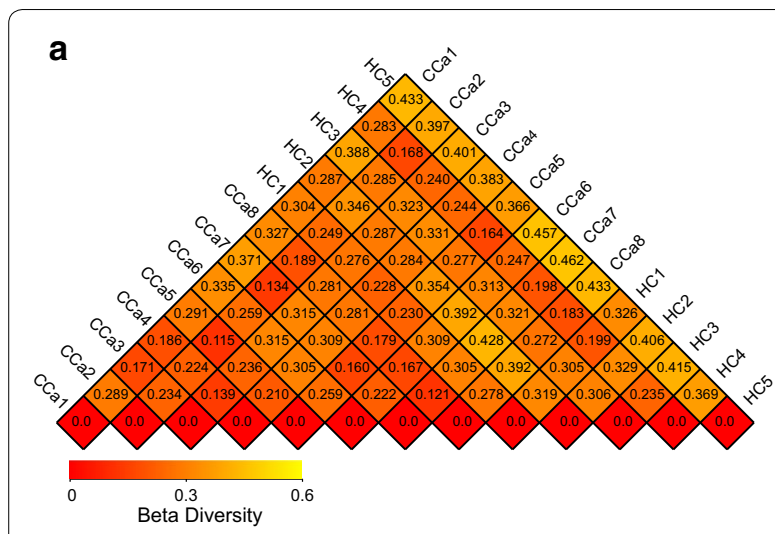

d

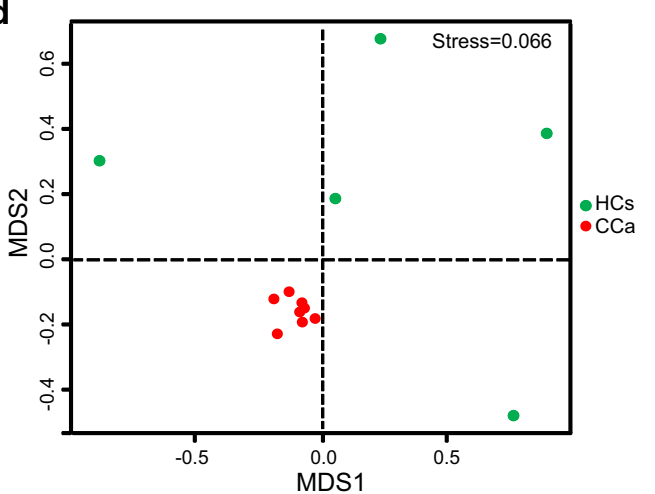

b

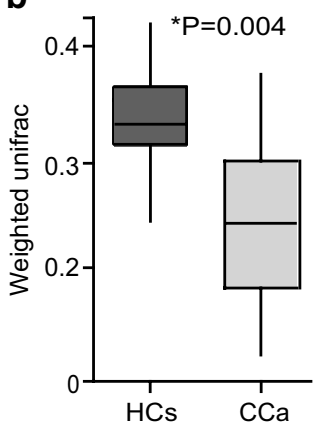

e

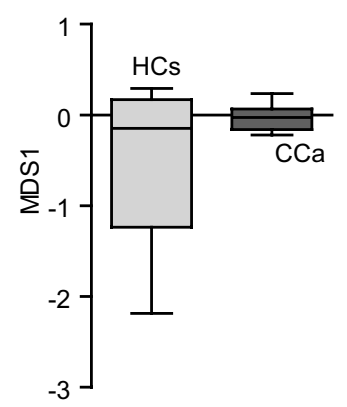

C

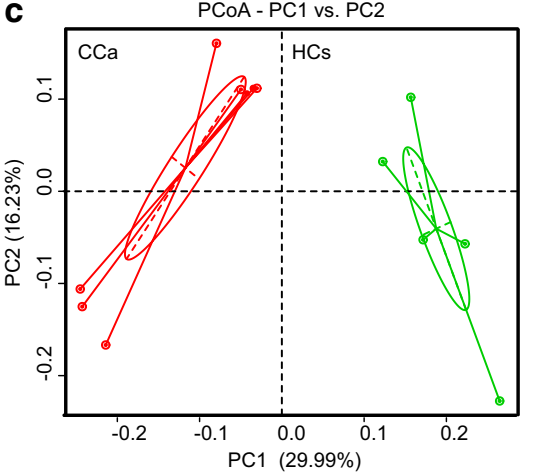

f

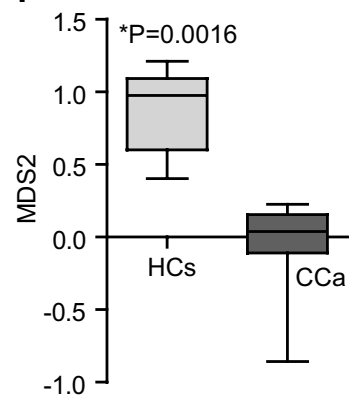

Fig. 2 Analysis of beta diversity in patient with cervical cancer compared with healthy controls. a Heatmap and $\mathbf{b}$ histogram of beta diversity based of Unifrac distance matrices ( $P=0.004$ by t-test). c PCoA analysis based of Unifrac distance matrices. Each sphere represents one sample. Samples separate into two clusters. d NMDS plots. (Green healthy control samples, HC1-HC5; Red patient samples, CCa1-CCa8). e, f Differences across groups are established the first and second MDS (e, MDS1 and $\mathbf{f}$, MDS2) values. MDS1, P > 0.05; MDS2, $P=0.0016$ by Mann-Whitney $U$ test

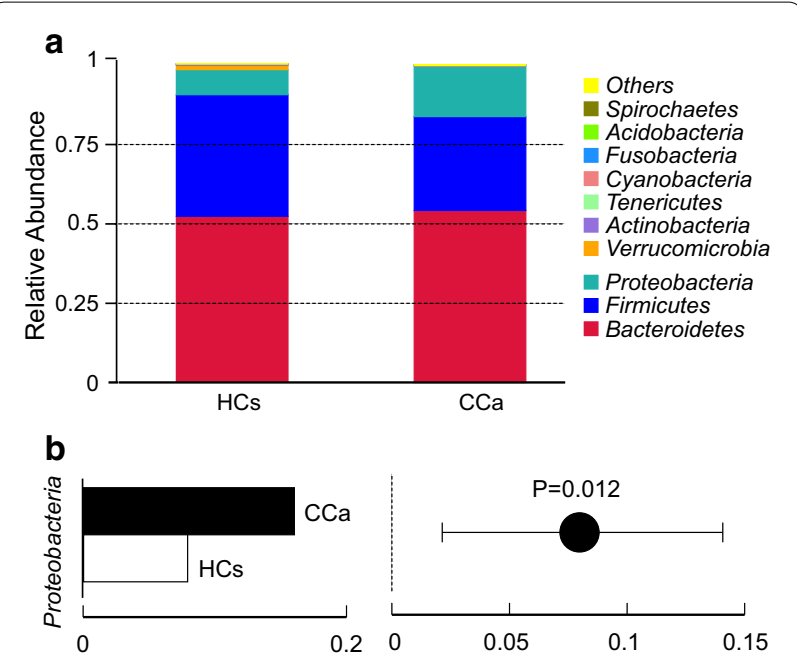

Fig. 3 Composition of gut microbiota in patient with cervical cancer on the phylum level. a The relative abundance of bacteria in the stool sample from the two groups. $\mathbf{b}$ The relative abundance of Proteobacteria was significantly higher in patients compared with controls $(P=0.012)$
A cladogram shown in Fig. 5b represented the connection between the significantly different taxa at different taxonomic levels. For example, Enterobacteriaceae (family) is under Enterobacteriales (order) which is under Gammaproteobacteria (class). A clade is a branch of organisms under a common ancestor (e.g. Proteobacteria is a common ancestor for all the genera belong to it). The significantly different taxa was shown in a tree like structure. Moreover, LDA score demonstrated that these differentially abundant taxa can be considered as potential biomarkers (LDA score $>4.0, \rho<0.05$ ).

\section{Discussion}

Cervical cancer is a heterogeneous and multifactorial disease, impacted by several different genetic and environmental factors. Although infection with HPV as a prerequisite for cervical cancer has been conclusively proven, the specific impact of other factors on this disease process is not yet well-characterized. In this study, we identified for the first time the significant alterations in gut microbial composition following cervical cancer 


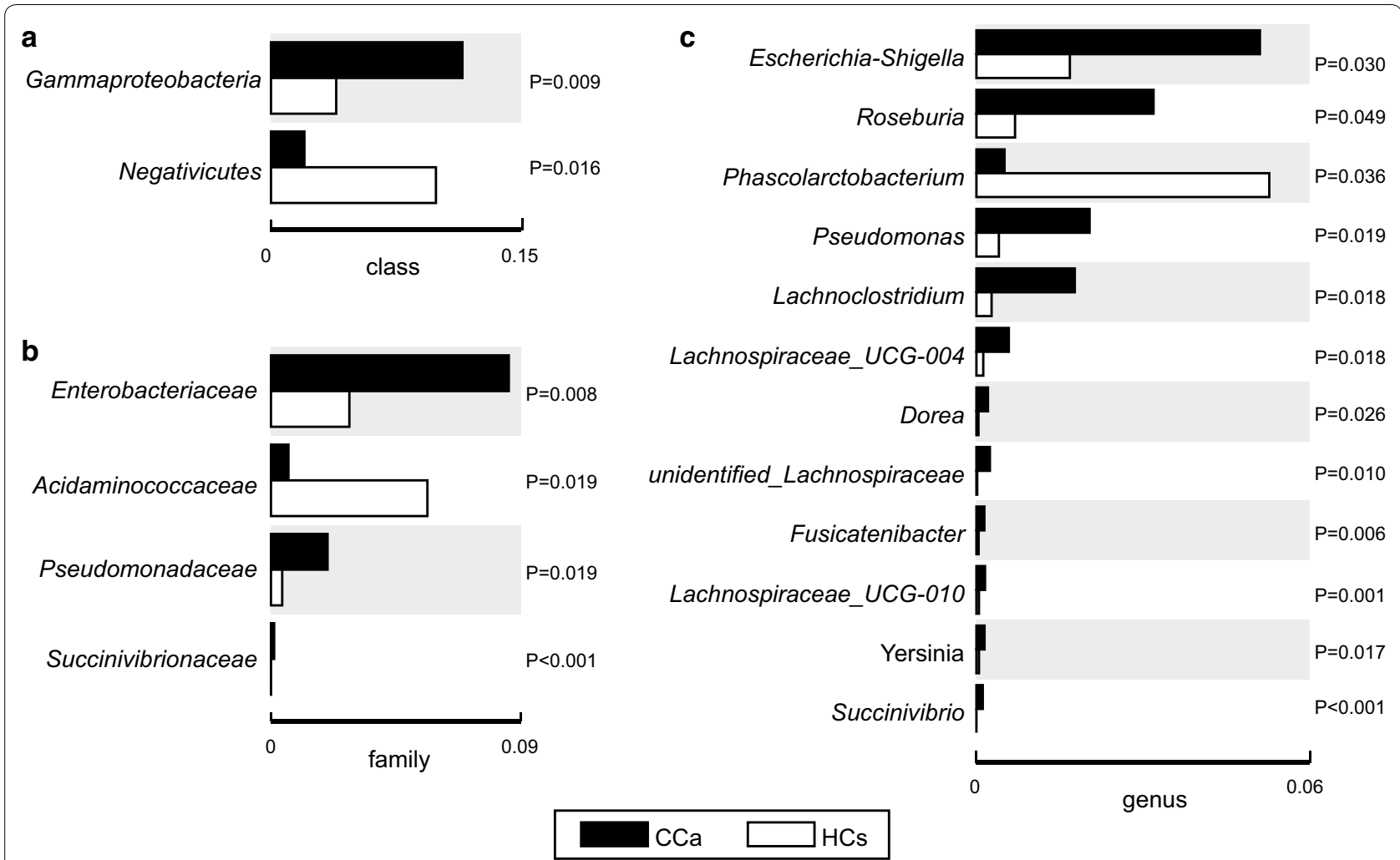

Fig. 4 Boxplots representing the average proportion of each 165 sequence read attributed to each taxon. $\mathbf{a}$ on the class level, b on the family level, c on the genus level (White healthy control samples, Black patient samples)

Table 3 Genera differences between patients and controls

\begin{tabular}{|c|c|c|c|}
\hline Genus & $\mathrm{HC}$ (mean) & $\mathrm{CCa}$ (mean) & P value* \\
\hline \multicolumn{4}{|l|}{ Higher in $\mathrm{HC}$ group } \\
\hline Phascolarctobacterium & 0.052614 & 0.005016 & 0.036 \\
\hline Halomonas & 0.000244 & 0.000932 & 0.003 \\
\hline \multicolumn{4}{|l|}{ Higher in CCa group } \\
\hline Succinivibrio & $0.0066 \times 10^{-3}$ & 0.001134 & 0.000 \\
\hline Ruminococcus & 0.000422 & 0.007318 & 0.019 \\
\hline Morganella & $0.00198 \times 10^{-2}$ & 0.000507 & 0.019 \\
\hline Shewanella & $0.0066 \times 10^{-2}$ & 0.000285 & 0.002 \\
\hline Proteus & $0.0066 \times 10^{-3}$ & 0.000132 & 0.005 \\
\hline Dorea & 0.000323 & 0.001902 & 0.006 \\
\hline
\end{tabular}

*By non-parametric $t$-test

development and evaluated the role of gut microbiota in the pathogenesis of cervical cancer. We showed that (i) there was a trend towards increased diversity within samples in the cervical cancer patients, and an obvious situation that gut microbiota community segregated between the CCa group and HC group, (ii) the gut microbial composition differs significantly in patients with cervical cancer compared to healthy subjects, and (iii) Bacteroidetes were the most predominant abundant bacterial taxa in the cervical cancer fecal specimens, while Firmicutes presented a relatively strikingly low abundance. Further LEfSe analysis indicated that gut microbiota taxa present could be used to differentiate cervical cancer patients from controls and thus could be regarded as potential biomarkers of clinical relevance. Taken together, this study demonstrates that patients with cervical cancer have their unique characteristic gut microbiota.

The connection of gut microbiota and both digestive diseases (Pascal et al. 2017; Wong et al. 2017; Heidrich et al. 2018) and non-digestive diseases (Scher and Abramson 2011; Dinan and Cryan 2017; Jie et al. 2017) have been suggested by numerous studies. However, most previously published studies were based on low throughput methods, such as traditional microbial culture, real-time fluorescent quantitative PCR and $16 \mathrm{~S}$ rRNA amplicon denaturing gradient gel electrophoresis. The results revealed only a small fraction of the overall microorganisms. Therefore, the overall picture of intestinal microbial communities and the corresponding microbial ecology are far from well understood. Over the past few years, the development and increased availability of next generation sequencing technologies, including 



Fig. 5 Taxonomic differences were detected between cervical cancer and healthy controls. a Linear discriminative analysis (LDA) effect size (LEfSe) analysis between healthy controls (HCs, red) and patients (CCa, green). b Cladogram showing differentially abundant taxonomic clades with an LDA score $>4.0$ among patients and controls

high-throughput 16S rRNA gene sequencing, has facilitated our understanding, and enabled astonishing discoveries about the microbial gene repertoire. Bacterial $16 \mathrm{~S}$ rRNA genes generally contain nine "hypervariable regions" (V1-V9) which represent considerable sequence diversity among different bacteria. Studies have isolated those sequences that identify a single bacterial species or differentiate between a limited number of different species (Stohr et al. 2005; Chakravorty et al. 2007). In this study, we examined bacterial taxonomic composition and phylogenetic diversity by PCR amplification of the $16 \mathrm{~S}$ rRNA V4 variable region and deep sequencing on the Illumina ${ }^{\circledR}$ HiSeq platform. The results were in consistent with other microbiome studies. Altered microbial diversity was observed in the fecal communities of cervical cancer patients, and they have overabundance of the genera indicated. On the other hand, data from different studies are scattered as proposed by Li et al. (2014). There is no comprehensive and uniformly processed database that represents the human gut microbiota worldwide; and moreover, it is also not clear at what pace the number of species and genes will continue to grow, with the increasing amount of sequencing data.

Cervical lesions are always linked to abnormal vaginal microbiota. Persistent infection with high-risk HPV is directly involved in the tumorigenesis of approximately $70 \%$ cases of cervical cancer (Ramakrishnan et al. 2015). HPV-positive women had vaginal microbiomes with greater bacterial diversity, including specifically being abundant in L. gasseri and G. vaginalis (Gao et al. 2013). Greater number of L. gasseri was associated with rapid remission of HPV (Brotman et al. 2014), and therefore a low risk of developing HPVassociated malignant transformation. However there are few studies of gut microbiota and cervical cancer. This study is, to our knowledge, the first time that the gut microbiota of cervical cancer patients have been analyzed by comprehensive next-generation sequencing, independent of culture methods. Patients with cervical cancer presented with a distinct composition of gut microbiota compared to healthy subjects, and the gut microbial communities may play a role in promoting cervical tumorigenesis. However, the exact mechanism is unclear. Firstly, bacterial microbiomeinduced tumorigenesis is thought to be associated with inflammatory response mediated by microorganismassociated molecular patterns (MAMP) and their activation of pattern recognition receptors (PRRs). Upon a MAMP binding to a PRR (such as Toll-like receptor), transcription of antibacterial proteins was stimulated by intracellular signaling cascade in the host epithelial cell. And pro-inflammatory cytokines including IL-17, TNF- $\alpha$, and IFN- $\gamma$ was also upregulated (CerfBensussan and Gaboriau-Routhiau 2010). This inflammatory response occurs not only locally, but more importantly at a systemic level, and thereby increases the risk of inflammation at distant sites (van der Meulen et al. 2016). Besides, gut microbiota modulate the enterohepatic circulation of estrogens, which circulate to exert effects on target organs like breast and uterine cervix (Goedert et al. 2015; van der Meulen et al. 2016). Chung's mouse studies have provided strong evidence 
that estrogen contributes to cervical carcinogenesis (Chung et al. 2010). Epidemiological data also indicated that women with the highest levels of circulating estrogen are at increased risk of developing cervical cancer (Chung et al. 2010). Otherwise, the gut microbial differences might affect cervical cancer risk through many other pathways, and further biofunctional studies are needed.

There are some limitations of this study. The number of enrolled patients in each subgroup was relatively small. Eight patients and five controls were collected, and thus we are unable to definitively distinguish between different stages of cancer and precancerous changes. Secondly, the risk factors, such as persistent positive HPV and many sexual partners were not measured. The association of risk factors and gut bacteria was not analyzed as well. Moreover, functional roles of identified bacterial species and interrelation to tumorigenesis of cervical cancer remain unclear.

In conclusion, we reported the comprehensive analysis of gut microbiota in patients with cervical cancer using a relatively small stool samples. Such analysis for diagnosis, prediction the risk of recurrence, and prevention using probiotics or antibiotics should be assessed in the future.

\begin{abstract}
Abbreviations
ACE: abundance-based coverage estimator; CCa: cervical cancer; HC: healthy control; LDA: linear discriminant analysis; LEfSe: discriminant analysis effect size; OTUs: operational taxonomic units; PCoA: principal coordinate analysis; PD: phylogenetic diversity.
\end{abstract}

\section{Authors' contributions}

ZQW and ZYY designed the study. ZQW, QXW, JZ, LLG and YZ performed the actual laboratory analyses. QXW, XW, JZ, LLG and YZ obtained the samples and analyzed the data. ZQW and ZYY wrote the manuscript. All authors read and approved the final manuscript.

\section{Author details \\ ${ }^{1}$ Department of Radiation Oncology, Key Laboratory of Cancer Prevention and Therapy, Tianjin's Clinical Research Center for Cancer, Tianjin Medical University Cancer Institute \& Hospital, National Clinical Research Center for Cancer, West Huanhu Road, West River District, Tianjin 300060, China. 2 Department of Gastroenterology, Nanjing First Hospital, Nanjing Medical University, 300 Guangzhou Road, Nanjing 210029, China. ${ }^{3}$ Department of Gas- trointestinal Oncology, Key Laboratory of Cancer Prevention and Therapy, Tianjin's Clinical Research Center for Cancer, Tianjin Medical University Cancer Institute \& Hospital, National Clinical Research Center for Cancer, West Huanhu Road, West River District, Tianjin 300060, China.}

\section{Acknowledgements}

Not applicable.

\section{Competing interests}

The authors declare that they have no competing interests.

\section{Availability of data and materials}

Data will be made available through publication and SRA database (PRJNA524816).

\section{Consent for publication}

Not applicable.

\section{Ethics approval and consent to participate}

The study was conducted according to the principles expressed in the Declaration of Helsinki and prior approval was obtained from the Medical Ethics Committee of Tianjin Medical University Cancer Institute \& Hospital. Informed consent was obtained from all patients.

\section{Funding}

This work was supported by the National Natural Science Foundation of China (81602677 to ZQW, 8170031045 to YZ).

\section{Publisher's Note}

Springer Nature remains neutral with regard to jurisdictional claims in published maps and institutional affiliations.

Received: 27 January 2019 Accepted: 18 March 2019

Published online: 23 March 2019

\section{References}

Basu P, Mittal S, Bhadra Vale D, Chami Kharaji Y (2017) Secondary prevention of cervical cancer. Best Pract Res Clin Obstet Gynaecol 47:73-85

Brotman RM, Shardell MD, Gajer P, Tracy JK, Zenilman JM, Ravel J, Gravitt PE (2014) Interplay between the temporal dynamics of the vaginal microbiota and human papillomavirus detection. J Infect Dis 210:1723-1733

Caporaso JG, Lauber CL, Walters WA, Berg-Lyons D, Lozupone CA, Turnbaugh PJ, Fierer N, Knight R (2011) Global patterns of 16 S rRNA diversity at a depth of millions of sequences per sample. Proc Natl Acad Sci USA 108(Suppl 1):4516-4522

Caporaso JG, Kuczynski J, Stombaugh J, Bittinger K, Bushman FD, Costello EK, Fierer N, Peña AG, Goodrich JK, Gordon Jl, Huttley GA, Kelley ST, Knights D, Koenig JE, Ley RE, Lozupone CA, McDonald D, Muegge BD, Pirrung M, Reeder J, Sevinsky JR, Turnbaugh PJ, Walters WA, Widmann J, Yatsunenko T, Zaneveld J, Knight R (2017) QIIME allows analysis of high-throughput community sequencing data. Nat Methods 7:335-336

Cerf-Bensussan N, Gaboriau-Routhiau V (2010) The immune system and the gut microbiota: friends or foes? Nat Rev Immunol 10:735-744

Chakravorty S, Helb D, Burday M, Connell N, Alland D (2007) A detailed analysis of $16 \mathrm{~S}$ ribosomal RNA gene segments for the diagnosis of pathogenic bacteria. J Microbiol Methods 69:330-339

Chao A (1987) Estimating the population size for capture-recapture data with unequal catchability. Biometrics 43:783-791

Chung SH, Franceschi S, Lambert PF (2010) Estrogen and ERalpha: culprits in cervical cancer? Trends Endocrinol Metab 21:504-511

DeSantis TZ, Hugenholtz P, Larsen N, Rojas M, Brodie EL, Keller K, Huber T, Dalevi D, Hu P, Andersen GL (2006) Greengenes, a chimera-checked $16 \mathrm{~S}$ rRNA gene database and workbench compatible with ARB. Appl Environ Microbiol 72:5069-5072

Dinan TG, Cryan JF (2017) Gut instincts: microbiota as a key regulator of brain development, ageing and neurodegeneration. J Physiol 595:489-503

Edgar RC (2004) MUSCLE: multiple sequence alignment with high accuracy and high throughput. Nucleic Acids Res 32:1792-1797

Edgar RC (2013) UPARSE: highly accurate OTU sequences from microbial amplicon reads. Nat Methods 10:996-998

Edgar RC, Haas BJ, Clemente JC, Quince C, Knight R (2011) UCHIME improves sensitivity and speed of chimera detection. Bioinformatics 27:2194-2200

Gao W, Weng J, Gao Y, Chen X (2013) Comparison of the vaginal microbiota diversity of women with and without human papillomavirus infection: a cross-sectional study. BMC Infect Dis 13:271

Goedert J, Jones G, Hua X, Xu X, Yu G, Flores R, Falk RT, Gail MH, Shi J, Ravel $J$, Feigelson HS (2015) Investigation of the association between the fecal microbiota and breast cancer in postmenopausal women: a population-based case-control pilot study. J Natl Cancer Inst. https://doi. org/10.1093/jnci/djv147

Guinane CM, Tadrous A, Fouhy F, Ryan CA, Dempsey EM, Murphy B, Andrews E, Cotter PD, Stanton C, Ross RP (2013) Microbial composition of human appendices from patients following appendectomy. MBio 4:e00366-12

Haas BJ, Gevers D, Earl AM, Feldgarden M, Ward DV, Giannoukos G, Ciulla D, Tabbaa D, Highlander SK, Sodergren E, Methé B, DeSantis TZ, Human Microbiome Consortium, Petrosino JF, Knight R, Birren BW (2011) 
Chimeric 16S rRNA sequence formation and detection in Sanger and 454-pyrosequenced PCR amplicons. Genome Res 21:494-504

Heidrich B, Vital M, Plumeier I, Döscher N, Kahl S, Kirschner J, Ziegert S, Solbach P, Lenzen H, Potthoff A, Manns MP, Wedemeyer H, Pieper DH (2018) Intestinal microbiota in patients with chronic hepatitis $\mathrm{C}$ with and without cirrhosis compared with healthy controls. Liver Int 38:50-58

Human microbiome project consortium (2012) A framework for human microbiome research. Nature 486:215-221

Jie Z, Xia H, Zhong SL, Feng Q, Li S, Liang S, Zhong H, Liu Z, Gao Y, Zhao H, Zhang D, Su Z, Fang Z, Lan Z, Li J, Xiao L, Li J, Li R, Li X, Li F, Ren H, Huang Y, Peng Y, Li G, Wen B, Dong B, Chen JY, Geng QS, Zhang ZW, Yang H, Wang J, Wang J, Zhang X, Madsen L, Brix S, Ning G, Xu X, Liu X, Hou Y, Jia H, He K, Kristiansen $\mathrm{K}$ (2017) The gut microbiome in atherosclerotic cardiovascular disease. Nat Commun 8:845

Kozik AJ, Nakatsu CH, Chun H, Jones-Hall YL (2017) Age, sex, and TNF associated differences in the gut microbiota of mice and their impact on acute TNBS colitis. Exp Mol Pathol 103:311-319

Li J, Jia H, Cai X, Zhong H, Feng Q, Sunagawa S, Arumugam M, Kultima JR, Prifti E, Nielsen T, Juncker AS, Manichanh C, Chen B, Zhang W, Levenez F, Wang J, Xu X, Xiao L, Liang S, Zhang D, Zhang Z, Chen W, Zhao H, Al-Aama JY, Edris S, Yang H, Wang J, Hansen T, Nielsen HB, Brunak S, Kristiansen K, Guarner F, Pedersen O, Doré J, Ehrlich SD, MetaHIT Consortium, Bork P, Wang J (2014) An integrated catalog of reference genes in the human gut micobiome. Nat Biotechnol 32:834-841

Magoč T, Salzberg SL (2011) FLASH: fast length adjustment of short reads to improve genome assemblies. Bioinformatics 27:2957-2963

Martin CM, Kehoe L, Spillane CO, O'Leary JJ (2007) Gene discovery in cervical cancer: towards diagnostic and therapeutic biomarkers. Mol Diagn Ther 11:277-290

Partida-Rodríguez O, Serrano-Vázquez A, Nieves-Ramírez ME, Moran P, Rojas L, Portillo T, González E, Hernández E, Finlay BB, Ximenez C (2017) Human intestinal microbiota: interaction between parasites and the host immune response. Arch Med Res 48(8):690-700

Pascal V, Pozuelo M, Borruel N, Casellas F, Campos D, Santiago A, Martinez X, Varela E, Sarrabayrouse G, Machiels K, Vermeire S, Sokol H, Guarner F, Manichanh C (2017) A microbial signature for Crohn's disease. Gut 66:813-822
Ramakrishnan S, Partricia S, Mathan G (2015) Overview of high-risk HPV's 16 and 18 infected cervical cancer: pathogenesis to prevention. Biomed Pharmacother 70:103-110

Scher JU, Abramson SB (2011) The microbiome and rheumatoid arthritis. Nat Rev Rheumatol 7:569-578

Segata N, Izard J, Waldron L, Gevers D, Miropolsky L, Garrett WS, Huttenhower C (2011) Metagenomic biomarker discovery and explanation. Genome Biol 12:R60

Sommer F, Bäckhed F (2013) The gut microbiota-masters of host development and physiology. Nat Rev Microbiol 11:227-238

Stohr K, Hafner B, Nolte O, Wolfrum J, Sauer M, Herten DP (2005) Species-specific identification of mycobacterial $16 \mathrm{~S}$ rRNA PCR amplicons using smart probes. Anal Chem 77:7195-7203

Ursell LK, Haiser HJ, Van Treuren W, Garg N, Reddivari L, Vanamala J, Dorrestein PC, Turnbaugh PJ, Knight R (2014) The intestinal metabolome: an intersection between microbiota and host. Gastroenterology 146:1470-1476

van der Meulen TA, Harmsen H, Bootsma H, Spijkervet F, Kroese F, Vissink A (2016) The microbiome-systemic disease connection. Oral Dis 22:719-734

Vrieze A, Nood EV, Holleman F, Salojärvi J, Kootte RS, Bartelsman JF, DallingaThie GM, Ackermans MT, Serlie MJ, Oozeer R, Derrien M, Druesne A, Van Hylckama Vlieg JE, Bloks VW, Groen AK, Heilig HG, Zoetendal EG, Stroes ES, de Vos WM, Hoekstra JB, Nieuwdorp M (2013) Transfer of intestinal microbiota from lean donors increases insulin sensitivity in individuals with metabolic syndrome. Gastroenterology 144(1):250

Wang M, Ahrné S, Jeppsson B, Molin G (2005) Comparison of bacterial diversity alone the human intestinal tract by direct cloning and sequencing of $16 \mathrm{~S}$ rRNA genes. FEMS Microbiol Ecol 54:219-231

Wang Q, Garrity GM, Tiedje JM, Cole JR (2007) Naive Bayesian classifier for rapid assignment of rRNA sequences into the new bacterial taxonomy. Appl Environ Microbiol 73(16):5261-5267

Wong SH, Zhao L, Zhang X, Nakatsu G, Han J, Xu W, Xiao X, Kwong TNY, Tsoi H, Wu WKK, Zeng B, Chan FKL, Sung JJY, Wei H, Wu J (2017) Gavage of fecal samples from patients with colorectal cancer promotes intestinal carcinogenesis in germ-free and conventional mice. Gastroenterology 153:1621-1633.e6

\section{Submit your manuscript to a SpringerOpen ${ }^{\odot}$ journal and benefit from:}

- Convenient online submission

- Rigorous peer review

- Open access: articles freely available online

- High visibility within the field

Retaining the copyright to your article

Submit your next manuscript at springeropen.com 